\title{
Reduced discomfort during high-definition transcutaneous stimulation using $6 \%$ benzocaine
}

\author{
Berkan Guleyupoglu ${ }^{1}$, Nicole Febles ${ }^{1}$, Preet Minhas ${ }^{1}$, Christoph Hahn ${ }^{2}$ and Marom Bikson ${ }^{1}$ \\ 1 Department of Biomedical Engineering, Neural Engineering Laboratory, The City College of the City University of New York, New York, NY, USA \\ ${ }^{2}$ Biomedical Engineering, University of Applied Sciences Hamburg, Hamburg, Germany
}

\author{
Edited by: \\ Stefano Ferraina, Sapienza \\ University of Rome, Italy \\ Reviewed by: \\ Andre R. Brunoni, Universidade de \\ São Paulo, Brazil \\ Maria Teresa Fiorenza, Sapienza \\ University of Rome, Italy \\ *Correspondence: \\ Berkan Guleyupoglu, Department of \\ Biomedical Engineering, Neural \\ Engineering Laboratory, The City \\ College of the City University of \\ New York, 160 Convent Ave., T-463 \\ Steinman Hall, New York, NY \\ 10031, USA \\ e-mail: bguleyup@gmail.com
}

Background: High-Definition transcranial Direct Current Stimulation (HD-tDCS) allows for non-invasive neuromodulation using an array of compact (approximately $1 \mathrm{~cm}^{2}$ contact area) "High-Definition" (HD) electrodes, as compared to conventional tDCS (which uses two large pads that are approximately $35 \mathrm{~cm}^{2}$ ). In a previous transcutaneous study, we developed and validated designs for HD electrodes that reduce discomfort over $>20$ min session with $2 \mathrm{~mA}$ electrode current.

Objective: The purpose of this study was to investigate the use of a chemical pretreatment with $6 \%$ benzocaine (topical numbing agent) to further reduce subjective discomfort during transcutaneous stimulation and to allow for better sham controlled studies.

Methods: Pre-treatment with $6 \%$ benzocaine was compared with control (no pretreatment) for 22 min $2 \mathrm{~mA}$ of stimulation, with either CCNY-4 or Lectron II electroconductive gel, for both cathodal and anodal transcutaneous (forearm) stimulation (eight different combinations).

Results: Results show that for all conditions and polarities tested, stimulation with HD electrodes is safe and well tolerated and that pretreatment further reduced subjective discomfort.

Conclusion: Pretreatment with a mild analgesic reduces discomfort during HD-tDCS.

Keywords: transcutaneous, tDCS, HD-tDCS, sensation, direct current stimulation

\section{INTRODUCTION}

Transcranial Direct Current Stimulation (tDCS) is an investigational neuromodulation technique that uses scalp electrodes to deliver 1-2 mA of direct current for 10-20 min (Guleyupoglu et al., 2013; Villamar et al., 2013). Whereas conventional tDCS uses two large pads (approximately $35 \mathrm{~cm}^{2}$ contact area, although not limited to this size), High-Definition tDCS (HD-tDCS) uses an array of smaller HD electrodes (approximately $1 \mathrm{~cm}^{2}$ contact area) to deliver current in a more target specific manner (Datta et al., 2008, 2009; Dmochowski et al., 2011; Guleyupoglu et al., 2013). Previously we showed that control over HD electrode parameters, namely shape, electrode material, and gel, allows for tolerated stimulation with no lasting skin irritation (Minhas et al., 2010). Several clinical studies have supported the safety and tolerability of HD-tDCS (Dundas et al., 2007; Nitsche et al., 2009; Borckardt et al., 2012; Caparelli-Daquer et al., 2012; Kuo et al., 2013); in some cases pre-treatment with mild topical anesthetic containing a low-concentration of benzocaine was used. Here we directly quantified if pre-treatment with a cream containing $6 \%$ benzocaine was effective in reducing discomfort across the forearm during $22 \mathrm{~min}$ of stimulation at $2 \mathrm{~mA}$. The forearm was used during the investigation as it provides a model that does not involve unintended direct stimulation of the brain. Though clinical trials support HD-tDCS as a well-tolerated technique, approaches to reduce discomfort and tingling during stimulation may further enhance tolerability and sham reliability.

\section{METHODS}

Ten healthy subjects, both male (5) and female (5) with ages ranging from 18 to 35, participated in this study. Each subject gave informed consent before being included in this study. This study was approved by the Institutional Review Board of The City College of New York. A total of eight different conditions were tested in this series using combinations of anodal/cathodal stimulation polarity, CCNY-4/Lectron electroconductive gel, and control/pretreatment conditions (e.g., anodal stimulation with CCNY-4 gel and no pretreatment). Each subject underwent stimulation under all eight conditions in random order and was blinded to the set-up in regards to anodal/cathodal and which electrogel was used. Stimulation was administered using a constant current stimulator (Schneider, Germany or Soterix Medical Inc. $1 \times 1$ tDCS, New York, NY); $2 \mathrm{~mA}$ of current was delivered to the forearm for $22 \mathrm{~min}(20 \mathrm{~min}$ stimulation, 2 min post-stimulation), with a $10 \mathrm{~s}$ ramp up before stimulation, and a $10 \mathrm{~s}$ ramp down before completion of the stimulation. $\mathrm{Ag} / \mathrm{AgCl}$ sintered ring electrodes were used (one active and two 
returns); the active electrode was positioned in the center of the forearm, and the two returns electrodes were positioned on the upper and lower forearm at approximately $5 \mathrm{~cm}$ apart, immersed in a high volume of gel - the use of two electrodes with high contact area resulted in sensation being restricted largely to the center active electrode; thus stimulation polarity, "anodal" or "cathodal" is used here in references to the center electrode polarity. The electrode-skin contact area was either pretreated with $0.2 \mathrm{ml}$ of $6 \%$ benzocaine (Lanacane brand) (and left on the skin before applying gel and stimulation for approximately $8 \mathrm{~min}$ ) or was left untreated (control). The sintered $\mathrm{Ag}-\mathrm{Ag} / \mathrm{Cl}$ electrodes (550025, Ring Electrode Stens Corp.) were held in place using electrode holders (Soterix Medical, New York, NY,
HD-1) and were immersed in either CCNY-4 (custom made) or Lectron (Lectron II, Pharmaceutical Innovation Inc., Newark, NJ, USA). Subjects were asked to rate the discomfort felt, using a pain scale from 0 to 10 ( 0 being no pain and 10 being the worst imaginable pain ever), after stimulation ramped up, every minute during stimulation and after stimulation ramped down. Subjects were also asked to describe the sensation that they were feeling ("burning", "prickling", etc.). Subjects were alerted when stimulation was initiated but not when stimulation terminated. After stimulation was terminated, a period of 1 week was used as a "wash-out" period prior to stimulating the subject again. To analyze the three independant factors (gel type, polarity, and whether or not pretreatment was applied)

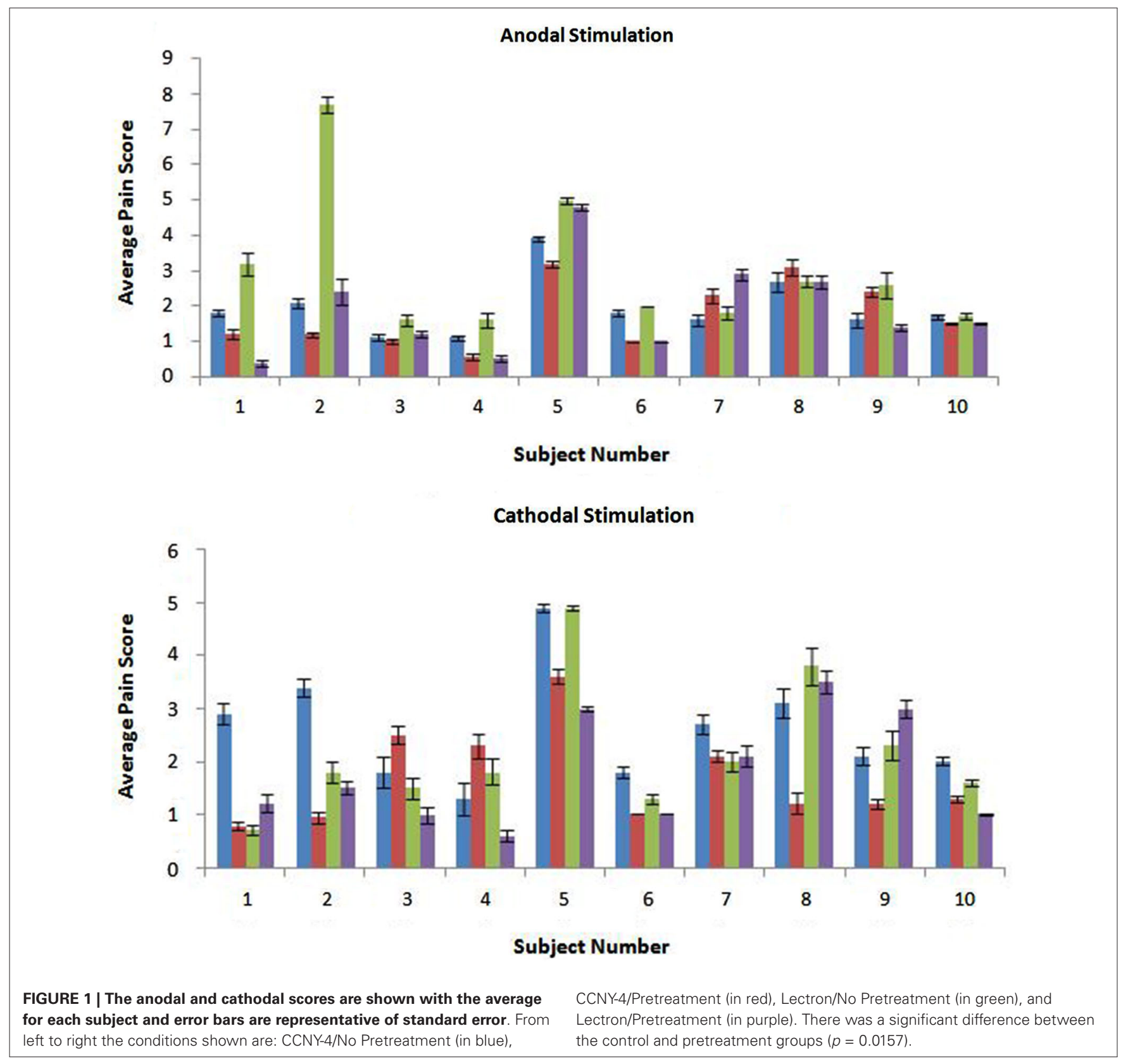


in this experiment, a 3-way repeated measures ANOVA was utilized.

\section{RESULTS}

Stimulation was well tolerated across subjects and conditions (Figure 1). Regardless of polarity and gel type, pretreatment reduced subject sensitivity to stimulation $(p=0.02, F=6.12)$. Further investigation into the effects of polarity $(p=0.80, F=$ $0.06)$ and gel type ( $p=0.45, F=0.58)$ show no significant difference on subject sensitivity to stimulation. The interaction effect between these three factors was also investigated. The interaction between polarity and gel type did not significantly reduce subject sensitivity to stimulation ( $p=0.16, F=1.97$ ) as well as the interaction between polarity and pretreatment $(p=0.96, F<$ $0.01)$. The interaction between gel type and pretreatment was also not significant in the reduction of sensitivity $(p=0.73, F=$ 0.12 ). Finally, the interaction between the three factors was not significant in the reduction of subject sensitivity to stimulation $(p=0.19, F=1.74)$.

\section{DISCUSSION}

The motivation behind this study was to reduce the level of discomfort that the subjects feel during stimulation. In this study, pretreatment with $6 \%$ benzocaine was shown to decrease discomfort for subjects during stimulation. Benzocaine was chosen for this investigation since it is a mild local anesthetic that is typically used as a pain reliever which could help reduce any discomfort that subjects may have during stimulation. The most common sensations felt by subjects were itching, tingling and prickling however, these sensations were not strictly correlated with the pain score reported. To assess the role of carrier and also electrode design, we evaluated the effect of aloe and hydrocortisone on discomfort. In the experiments we combined anode/cathode and considered two electrode designs (Tin and $\mathrm{Ag} / \mathrm{AgCl}$ ). In the experiments $(n=8)$ we observed no effect on discomfort by carrier for either electrode design $(p=0.18, p=0.25)$. Since there was no observed effect on discomfort by carrier, the control for this design with no pretreatment was used. However, it is still possible that there is a placebo effect present.

Current flow distribution and electrochemical changes are critically dependant on all aspects of electrode design (Dundas et al., 2007; Minhas et al., 2010; Kronberg and Bikson, 2012; Caytak et al., 2013). It is important to emphasize that the results of this study are specific to our validated HD electrode design (Minhas et al., 2010; Borckardt et al., 2012), and further more apply only to the dose tested (e.g., up to $22 \mathrm{~min}, 2 \mathrm{~mA}$, one session), such that, as with any treatment, adoption of new electrode designs, increased stimulation intensity, or repetition warrants explicit testing. A recent report showed no effect on sensation using lidocaine, an agent similar to benzocaine, when testing conventional tDCS electrodes-indicating the efficacy of anesthetic is dependent on electrode design or montage (Guarienti et al., 2014).

\section{REFERENCES}

Borckardt, J. J., Bikson, M., Frohman, H., Reeves, S. T., Datta, A., Bansal, V., et al. (2012). A pilot study of the tolerability and effects of high-definition transcranial direct current stimulation (HD-tDCS) on pain perception. J. Pain 13, 112-120. doi: 10.1016/j.jpain.2011.07.001

Caparelli-Daquer, E. M., Zimmermann, T. J., Mooshagian, E., Parra, L. C., Rice, J. K., Datta, A., et al. (2012). A pilot study on effects of $4 \times 1$ high-definition tDCS on motor cortex excitability. Conf. Proc. IEEE Eng. Med. Biol. Soc. 2012, 735-738. doi: 10.1109/EMBC.2012.6346036

Caytak, H. B., Batkin, I., Mekonnen, A., Shapiro, D., Hassouni, S., Li, G., et al. (2013). "Superabsorbent polymer electrode for transcranial direct current stimulation," in IEEE International Symposium on Medical Measurements and Applications Proceedings (MeMeA) (Piscataway, NJ: IEEE), 320-324.

Datta, A., Bansal, V., Diaz, J., Patel, J., Reato, D., and Bikson, M. (2009). Gyri-precise head model of transcranial direct current stimulation: improved spatial focality using a ring electrode versus conventional rectangular pad. Brain Stimul. 2, 201207. doi: 10.1016/j.brs.2009.03.005

Datta, A., Elwassif, M., Battaglia, F., and Bikson, M. (2008). Transcranial current stimulation focality using disc and ring electrode configurations: FEM analysis. J. Neural Eng. 5, 163-174. doi: 10.1088/1741-2560/5/2/007

Dmochowski, J. P., Datta, A., Bikson, M., Su, Y., and Parra, L. C. (2011). Optimized multi-electrode stimulation increases focality and intensity at target. J. Neural Eng. 8:046011. doi: 10.1088/1741-2560/8/4/046011

Dundas, J. E., Thickbroom, G. W., and Mastaglia, F. L. (2007). Perception of comfort during transcranial DC stimulation: effect of $\mathrm{NaCl}$ solution concentration applied to sponge electrodes. Clin. Neurophysiol. 118, 1166-1170. doi: 10.1016/j. clinph.2007.01.010

Guarienti, F., Shiozawa, P., Cordeiro, Q., Boggio, P. S., Bensenor, I. M., Lotufo, P. A., et al. (2014). Reducing transcranial direct current stimulation (tDCS)induced erythema with skin pretreatment: considerations for sham-controlled tDCS clinical trials. Neuromodulation in press.

Guleyupoglu, B., Schestatsky, P., Edwards, D., Fregni, F., and Bikson, M. (2013). Classification of methods in transcranial electrical stimulation (tES) and evolving strategy from historical approaches to contemporary innovations. J. Neurosci. Methods 219, 297-311. doi: 10.1016/j.jneumeth.2013.07.016

Kronberg, G., and Bikson, M. (2012). Electrode assembly design for transcranial direct current stimulation: a FEM modeling study. Conf. Proc. IEEE Eng. Med. Biol. Soc. 2012, 891-895. doi: 10.1109/EMBC.2012.6346075

Kuo, H. I., Bikson, M., Datta, A., Minhas, P., Paulus, W., Kuo, M. F., et al. (2013). Comparing cortical plasticity induced by conventional and high-definition $4 \times 1$ ring tDCS: a neurophysiological study. Brain Stimul. 6, 644-648. doi: 10.1016/j. brs.2012.09.010

Minhas, P., Bansal, V., Patel, J., Ho, J. S., Diaz, J., Datta, A., et al. (2010). Electrodes for high-definition transcutaneous DC stimulation for applications in drug delivery and electrotherapy, including tDCS. J. Neurosci. Methods 190, 188-197. doi: 10.1016/j.jneumeth.2010.05.007

Nitsche, M. A., Boggio, P. S., Fregni, F., and Pascual-Leone, A. (2009). Treatment of depression with transcranial direct current stimulation (tDCS): a review. Exp. Neurol. 219, 14-19. doi: 10.1016/j.expneurol.2009.03.038

Villamar, M. F., Wivatvongvana, P., Patumanond, J., Bikson, M., Truong, D. Q., Datta, A., et al. (2013). Focal modulation of the primary motor cortex in fibromyalgia using 4x1-ring high definition transcranial direct current stimulation (HD-tDCS): immediate and delayed analgesic effects of cathodal and anodal stimulation. J. Pain 14, 371-383. doi: 10.1016/j.jpain.2012. 12.007

Conflict of Interest Statement: Dr. Marom Bikson is the inventor of technologies dealing with transcranial electrical stimulation for which The City College of New York holds patents.

Received: 25 March 2014; accepted: 25 June 2014; published online: 11 July 2014. Citation: Guleyupoglu B, Febles N, Minhas P, Hahn C and Bikson M (2014) Reduced discomfort during high-definition transcutaneous stimulation using $6 \%$ benzocaine. Front. Neuroeng. 7:28. doi: 10.3389/fneng.2014.00028

This article was submitted to the journal Frontiers in Neuroengineering.

Copyright $\odot 2014$ Guleyupoglu, Febles, Minhas, Hahn and Bikson. This is an openaccess article distributed under the terms of the Creative Commons Attribution License (CC BY). The use, distribution or reproduction in other forums is permitted, provided the original author(s) or licensor are credited and that the original publication in this journal is cited, in accordance with accepted academic practice. No use, distribution or reproduction is permitted which does not comply with these terms. 